\title{
Musikindustrie und Urheberrechtsschutz im Internet
}

Einschneidende Veränderungen in Wirtschaft und Gesellschaft fangen häufig mit einer technischen Innovation an. Die Erfolgsgeschichte von InternetTauschbörsen ( $\mathrm{P}_{2} \mathrm{P}-\mathrm{Netzwerke}$ ) zeigt einmal mehr - angefangen mit Napster bis hin zu den heutigen Emule Mods - die Innovationsfähigkeit und Veränderungsbereitschaft der Gesellschaft hinsichtlich technischer Möglichkeiten. Mittlerweile finden sich mehr als 120 verschiedene $\mathrm{P}_{2} \mathrm{P}-\mathrm{Software}^{\mathrm{I}}$, die es Nutzern ermöglichen, urheberrechtlich geschützte Werke herunterzuladen. Alleine in Deutschland ist 2004 die Zahl der heruntergeladenen Filme auf I 4,6 Millionen gestiegen. ${ }^{2}$ Immer wieder werden auch Filme, wie z. B. der neue Star-Wars-Film »Die Rache der Sith «3, vor der eigentlichen Kinopremiere in P2P-Netzwerken veröffentlicht. Trotz des verbreiteten Optimismus in der Urheberindustrie ${ }^{4}$ nicht zuletzt bedingt durch den Start diverser legaler Musikangebote wie iTunes von Apples - bleibt der Austausch von Musik- und Filmdateien in $\mathrm{P}_{2} \mathrm{P}-\mathrm{Netz}-$ werken ein weiterhin akutes Problem ${ }^{6}$. Durch den I. Korb der Urheberrechtsreform $^{7}$ wurde der Urheberbranche erstmals die Möglichkeit eröffnet, gegen Nutzer von $\mathrm{P}_{2} \mathrm{P}-\mathrm{Netzwerken} \mathrm{effektiv} \mathrm{vorzugehen.}{ }^{8}$ Seit einiger Zeit versuchen daher insbesondere die Musikindustrie und ihre Verwertungsgesellschaften gegen illegales Filesharing vorzugehen. Auf diesem Weg scheint die Urheberindustrie ihrem Ziel nach Bekämpfung von Filesharing durch das jüngste Urteil des US Supreme Courts 9 ein Stück näher gekommen zu sein. In diesem Urteil wurde grundsätzlich festgestellt, dass Tauschbörsensoftware illegal sei. ${ }^{1 \circ}$ Welche Auswirkungen dieses amerikanische Urteil auf das Filesharing weltweit und auf das deutsche Rechtssystem hat, ist noch nicht abzusehen. Dennoch bleiben grundsätzliche Probleme trotz dieses Urteils bestehen.

\section{Probleme und Lösungsansätze}

Wer rechtlich gegen Filesharing vorgehen will, muss sich Interessenkonflikte, denen $\mathrm{P}_{2} \mathrm{P}-\mathrm{B}$ örsen unterworfen sind, vergegenwärtigen. Diese könnten unterschiedlicher nicht sein. Auf der einen Seite kämpft die Urheberindustrie (Her-

\footnotetext{
I CNet Download.com, Stand: 03.03.2005, http://www.download.com/MP3-Search-Tools/3 I 50-2 I66_4o.html?tag=dir

2 FFA Brenner Studie 2004, S. 23; IFPI Presseerklärung vom 01.10.2004, »Brennerstudie 2004, http:// www.ifpi.de/wirtschaft/wirtschaft-402.htm; Der Stern vom 27.10.2004, "Aufklärungsarbeit zeigt erste Wirkung «, http://www.stern.de/computer-technik/computer/?id=53 I6ro

3 Spiegel-Online vom 20.05.2004, »Neuer Star-Wars-Film schon im Web zu haben”, http://www.spiegel.de/netzwelt/netzkultur/0, is I $8,356703,00$.html

4 Spiegel-Online vom 27.01.2005, »Musikbranche wittert Morgenluft”, http://www.spiegel.de/netzwelt/ politik/0, I I I $8,338858,00 . \mathrm{html}$

s Spiegel-Online vom 23.I I.2004, "Apple-Aktie auf Vierjahreshoch”, http://www.spiegel.de/wirtschaft/ 0,15 I 8,3292 I $9,00 . h t m l$

6 FTD vom I 2.08.2004, »Exklusiv: Deutsche Musikindustrie bremst Talfahrt «, http://www.ftd.de/tm/me/ I092 I 7 I043435.html

7 Der I. Korb trat am I 3.09.2003 in Kraft

8 Schack, Urheber- und Urhebervertragsrecht, 2. Aufl. 200I, Rdn. 496; Harke, c't 5/2000, S. I I 2, »Musikkopien illegal?"; Erd, Online Recht kompakt, I. Aufl. 2000, S. 98; Kreutzer, GRUR 200I, I93 (201); Mönkemöller, GRUR 2000, 663 (667); Bosak, CR 200I, I76 (I8I); dagegen: Braun, GRUR 200I, I 106 ( 1 I 08 )

9 Urteil vom 27.6.2005; www.supremecourtus gov/opinions/o4pdf/04.480.pdf

Io Heise-Online vom $27.06 .2005 ; \mathrm{bttp} / /$ www. heise.de/newsticker/meldung/61 120
} 
steller von CD's und Filmen) um ihre Einkommensquelle, auf der anderen Seite stehen die Nutzer, die kostenloses und freies Entertainment wünschen. Auch Technologieunternehmen haben ein wirtschaftliches Interesse am Filesharing, wenn sie durch den Verkauf von Rohlingen, DSL-Internetverbindungen oder der Herstellung von Brennern und anderen Kopiergeräten von der Downloadund Burnmania leben. Neben diesen unterschiedlichen Interessen ist auch die Globalität des Mediums Internet ein Problem für die Bekämpfung des OnlineRaubkopierens. ${ }^{\text {II }}$ Ein Ausdruck dieser Problematik ist die immense Zahl der $\mathrm{P}_{2} \mathrm{P}$-Software, die dem Nutzer zur Verfügung steht. ${ }^{\mathrm{I2}}$ Man geht davon aus, dass es aktuell alleine 2,7 Millionen aktive Benutzer des eDonkey2000-Netzwerkes gibt. ${ }^{13}$ Trotz des Vorgehens gegen illegale Internet-Tauschbörsen von Seiten der Musik- und Filmindustrie in den USA ist die Zahl der illegalen Downloads um ca. $20 \%$ bis zum Jahre 2003 gestiegen. ${ }^{14}$ Im Juni 2003 wurden bereits mehr als 820 Millionen Musiktitel zum Kopieren angeboten, im Juni 2004 war die Milliarden-Grenze deutlich überschritten. Is Trotz verstärkter rechtlicher Sanktionen ist das Problem des Filesharing nach wie vor akut und bedarf zusätzlicher juristischer Anstrengungen.

Als Lösungsmöglichkeiten werden mehrere Ideen diskutiert, die sich in begleitende und aktive juristische Vorgehensweisen unterscheiden lassen. Als begleitende Lösungsmöglichkeiten wird u.a. das sog. Digital-Rights-Management (DRM) eingesetzt. ${ }^{16}$ Unter DRM versteht man den Einsatz von Kopierschutzmaßnahmen, die Downloads ohne Zustimmung des Urhebers unmöglich machen sollen. Auch haben sich in den letzten Jahren verstärkt legale Alternativen in Form von kostenpflichtigen Musikdownloads angeboten ${ }^{17}$, um überhaupt dem Nutzer eine Alternative zu bieten. ${ }^{18}$ Zunehmend wird auch versucht, mit Werbemaßnahmen, welche die Nutzer über mögliche rechtliche Konsequenzen im Zusammenhang mit Filesharing aufklären sollen, einen Sinneswandel bei Nutzern herbeizuführen. ${ }^{19}$

Juristisch wird gegen Filesharing erst seit der Umsetzung des I. Korbs der Urheberrechtsreform vorgegangen. Vorrangigste Aufgabe der Rechteinhaber ist die Identifizierung der Täter bzw. Nutzer, die gegen das Urheberrecht verstoßen. Erst durch die Identifizierung der Person, die hinter einer Internet-Adresse steht, kann eine Klage spezifiziert eingereicht werden. Gegenwärtig wird ein solcher Auskunftsanspruch im Zusammenhang mit dem 2. Korb der Urheber-

I I Heise-Online vom 22.03.2005, »Europaweiter Streit ums Urheberrecht«, http://www.heise.de/newsticker/meldung/57800; Heise-Online vom 07.03.2005, "Allofmp3.com schlüpft durch eine Gesetzeslücke«, http://www.heise.de/newsticker/meldung/57 I 57

I 2 War am Anfang "Napster « das einzigste Programm dieser Art, finden sich mittlerweile mehr als I 20 verschiedene P2P-Software. (Quelle: CNet Download.com, Stand: 03.03.2005, http://www.download.com/ $\mathrm{MP}_{3}$-Search-Tools/3 I 50-2 I66_4-0.html?tag=dir )

I 3 Wikipedia.org, http://de.wikipedia.org/wiki/Emule

I4 Heise-Online vom I 2.07.2004, »Online-Tauschbörsen sind beliebter denn je«, http://www.heise.de/ newsticker/meldung/49023

Is Heise-Online vom I 2.07.2004, »Online-Tauschbörsen sind beliebter denn je«, http://www.heise.de/ newsticker/meldung/ 49023

I6 Heise-Online vom I9.I 2.2004, "Sony BMG und EMI Music kooperieren bei digitalen Musikformaten«, http://www.heise.de/newsticker/meldung/54445; Heise-Online vom I8.1 2.2004, "Neuer Audio-CDKopierschutz für den USA-Markt«, http://www.heise.de/newsticker/meldung/54433; Heise-Online vom 09.03.2003, »Bill Gates: Palladium dient auch dem Urheberschutz", http://www.heise.de/newstik$\mathrm{ker} / \mathrm{meldung} / 35 \mathrm{I} 3 \mathrm{I}$

I7 Bsp.: www.musicload.de

I 8 Bis vor ca. 2 Jahren bestand noch keine Möglichkeit zum legalen Download von Musik

I9 Heise-Online vom 14.02.2003, "Medienindustrie warnt vor Sicherheitsrisiko Tauschbörse«, http:// www.heise.de/newsticker/meldung/34524, Heises Online vom 24.10.2002, »US-Unterhaltungsindustrie ruft Firmen zum Kampf gegen Piraterie auf \&, http: $/$ www heise.de/newsticker/meldung/3 1796 
rechtsreform, deren Zukunft durch den Regierungswechsel im Herbst 2005 ungewiss ist, diskutiert. Jedoch gibt es einige Stimmen, welche de lege lata schon einen zivilrechtlichen Drittauskunftsanspruch gegenüber Access-Providern (den Zugangsvermittlern das Datenaustauschs) zu begründen versuchen. Ebenso wird ein Vorgehen gegen Hersteller und Vertreiber von $\mathrm{P}_{2} \mathrm{P}$-Software erörtert. Diese Argumentationen sollen näher beleuchtet werden.

\section{Zivilrechtlicher Drittauskunftsanspruch gegenüber Access-Providern}

Ein Auskunftsanspruch gegenüber Access-Providern über die Nutzer von $\mathrm{P}_{2} \mathrm{P}-$ Netzwerken ist Voraussetzung für die rechtliche Verfolgung von »illegalen« Internet-Tauschbörsen. Nur wenn dem Nutzungsrechtsinhaber der nichtgewerbliche Nutzer namentlich mit einer zustellungsfähigen Adresse bekannt ist, wird ein rechtliches Vorgehen möglich. Hinsichtlich des Auskunftsanspruches wird die Anwendbarkeit des im Urheberrecht normierten Anspruches nach \ Iог a UrhG diskutiert. Eine unmittelbare Anwendung des $\$ Iora UrhG im Hinblick auf Drittauskunftsansprüche gegenüber Access-Providern kann nach unstrittiger Meinung ${ }^{20}$ jedoch nicht begründet werden. Festgestellt werden kann, dass aufgrund des Nicht-Bestehens einer planwidrigen Regelungslücke bzw. mangelnden Ähnlichkeit der Interessenslage weder eine direkte Anwendung des $\$ rora UrhG noch eine analoge Anwendung plausibel erscheint. Dies ergibt sich zum einen daraus, dass die Regelung sich nur auf gewerbliche Vertriebswege bzw. Quellen beschränkt ${ }^{21}$ und nicht ein generelles Auskunftsrecht ist, und zum anderen, dass vieles für ein bewusstes Weglassen eines weitergehenden Auskunftsanspruches gegenüber Dritten durch den Gesetzgeber spricht. ${ }^{22}$

Wenn ein Urheberrechtsinhaber von dem Access-Provider keine Auskunft erhalten kann, dann wäre es vorstellbar, dass er direkt vom Provider als Mitstörer Schadensersatz erhalten kann. Es ist nicht möglich, eine Mitstörerhaftung zu begründen, es sei denn, ab der positiven Kenntnis des Access-Providers, was jedoch in keinem Fall zu einer Auskunftsverpflichtung Dritter führen kann. Sollte jedoch der Auskunftsanspruch aufgrund der Mitstörerhaftung bejaht werden, müsste ebenfalls der Gesichtspunkt der Zumutbarkeit bzgl. der Auskunftserteilung für den Access-Provider diskutiert werden.. ${ }^{23} \mathrm{Da}$ dies ebenfalls einen gesteigerten Aufwand zur Folge hätte, ist die Zumutbarkeit mehr als fragwürdig.

Zusätzlich zu diesen Ergebnissen können gegen einen Auskunftsanspruch des Urheberechtsinhabers nach $\mathbb{S}$ iora UrhG gegen den Access-Provider auch datenschutzrechtliche oder fernmelderechtliche Einwände geltend gemacht werden. 
Der Access-Provider unterliegt meiner Meinung nach sowohl den Regelungen als Telediensteanbieter sowie den Bestimmungen als Telekommunikationsdienst. $^{24}$

Der Access-Provider unterliegt sowohl den datenschutzrechtlichen Regelungen als Telediensteanbieter sowie als Telekommunikationsanbieter. Eine Herausgabe von personenbezogenen Daten im Zusammenhang mit dem Auskunftsanspruch gem. \iоra UrhG kommt aus Datenschutzgründen, aufgrund mangelnder Ermächtigung bzw. Vorrang des TDDSG und TDSV vor dem BDSG, nicht in Betracht. Die Herausgabe von personenbezogenen Daten wäre auch ein Verstoß gegen das Fernmeldegeheimnis, was für den Access-Provider u.U. sogar strafrechtliche Konsequenzen hätte.

\section{Maßnabmen gegen Hersteller und Vertreiber von $P_{2} P$-Software}

Neben der möglichen Hauptwaffe des zivilrechtlichen Auskunftsanspruches stehen noch besonders die Möglichkeiten des Vorgehens gegen die Hersteller und Vertreiber von P 2 P-Software zur Verfügung. »Was nicht da ist, kann auch keinen Schaden verursachen «, so dachten und denken vermutlich die Verwertungsgesellschaften über illegale Tauschbörsen. Doch ob diese »illegalen « Tauschbörsen auch wirklich »illegal « im juristischen Sinne sind, bleibt zu überprüfen. Eine der Möglichkeiten zur Bekämpfung des $\mathrm{P}_{2} \mathrm{P}-\mathrm{Problems} \mathrm{sind} \mathrm{mögli-}$ che Haftungs- und Unterlassungsansprüche gegen die Betreiber von Netzwerken bzw. Hersteller der Software. Besonders in den USA fanden solche Verfahren gegen zentrale Filesharing-Netzwerke statt. ${ }^{25}$ Festzuhalten bleibt, dass zentrale Systeme bzw. Tauschbörsen der Anwendbarkeit des TDG unterliegen. ${ }^{26}$ Dadurch begründet sich spätestens ab Kenntniserlangung und NichtErgreifung von Maßnahmen eine Störerhaftung. Folglich kann auch eine Sperrung bzw. Verwendung von Filtern bei zentralen Systemen verlangt werden. ${ }^{27}$ Eine Strafbarkeit wegen Beihilfe kommt ggf. auch in Frage. ${ }^{28}$ Bei dezentralen Systemen scheidet dagegen regelmäßig die Anwendbarkeit des TDG aus.29 Ebenfalls kann kein Haftungs- oder Unterlassungsanspruch bzgl. der Herstellung der Software begründet werden. Eine mögliche Lösung für die aktuelle Problematik der dezentralen Systeme kann das UWG bieten, da zumindest der Vertrieb von $\mathrm{P}_{2} \mathrm{P}-\mathrm{Software}$, nach der hier vertretenen Meinung, durch einen Unterlassungsanspruch verhindert werden kann..$^{\circ}$ Inwieweit dieser grundsätzlich mögliche Unterlassungsanspruch in der Praxis erfolgreich ist, bleibt abzu-

24 Zur Diskussion: Gegen die Einordnung als Teledienst: Hoeren/Sieber, Handbuch Multimediarecht 2003, Teil I6, Rdn. 32; Stadler, MMR 2002, 343 (344); Schmitz, MMR 200 I, I 50; RegTP, MMR I999, 557 (565); König/Lötz, CR 1999, 438 (444); Dietz/ Richter, CR I998, 528 (530); Felixberger, CR I998, I 43 (I 44); für die Einordnung als Teledienst: Schaar, Datenschutz im Internet, I. Aufl. 2002, Rdn. 262; ULD, MMR 2003, VII; Reg.Präs. Darmstadt, MMR 2003, 2 I 3; zur Diskussion: Spindler/Volkmann, K \& R 2002, 395 (399); Freytag, CR 2000, 600 (606)

25 U.a. die Urteile gegen Napster, Aimster und Audiogalaxy, siehe z.B.: U.S. District Court for the Northern District of California, Entscheidung vom 05.05.2000-(AEM Records Inc. v. Napster Inc.) $=G R U-$ RInt 2000, 1066; U.S. Court of Appeals for the Ninth Circuit, Entscheidung vom I2. 02. 200I - (AEM Records, Inc. et al. v. Napster, Inc.) = GRURInt 200I, 355 = GRURInt 200I, 273

26 Braun, GRUR 200I, I IO6 (I I I0); vgl. LG München I, ZUM 2000, 4I 8 (423), Schricker, Urheberrecht, 2.Aufl. I999, \97 Rdn. 40a; Möbring/Nicolini, UrhG, 2.Aufl. 2000, $\$ 97$ Rdn. 26; Kreutzer, GRUR $200 \mathrm{I}$

27 Siehe u.a. Braun, GRUR 200I, I I06 (I I IO)

28 Heghmanns, MMR 2004, I4

29 Keine Betreiber i.S.d. TDG, siehe: Braun, GRUR 200 I, I I 06 (I I I0)

30 Auf Grundlage der Argumentation Vortei1 durch-Rechtsbrach, siehe: LG Frankfurt/Main, NJWEWettbR I 998, 2.7 I 
warten, da es durch die Internationalität des Internet u.U. zu Schwierigkeiten bzgl. der Zuständigkeit und Anwendung des deutschen Rechtes kommen könnte. Zumindest lassen sich jedoch der Vertrieb von P2P-Software, welche zum Download von Anbietern, wie z.B. Chip-Online ${ }^{3}$, die ihren Sitz in Deutschland haben, angeboten werden, verhindern. Zusammengefaßt bedeutet dies, dass »illegale « Internet-Tauschbörsen nach deutschem Recht, anders als das jüngste Urteil in den $\mathrm{USA}^{32}$, an sich »legal « sind. In die gleiche Richtung der hier vertretenen Auffassung geht das aktuelle Gerichtsverfahren von »Premiere « gegen »Cybersky «33, welches vermutlich ebenfalls erhebliche Auswirkungen auf die Zulässigkeit von $\mathrm{P}_{2} \mathrm{P}$-Software bzw. Tauschbörsen haben dürfte. 34

\section{Fazit}

Die Überprüfungen der Möglichkeiten für ein Drittauskunftsrecht hat gezeigt, dass de lege lata nur der Umweg über die strafrechtlichen Vorschriften zu dem gewünschten Ergebnis gelangt. Auch die Maßnahmen gegen die Hersteller und Vertreiber von $\mathrm{P}_{2} \mathrm{P}$-Software sind für die Urheberrechtsindustrie nicht befriedigend. Es bleibt daher abzuwarten, welche Entwicklungen in der Musikindustrie und ein betreffender Urheberrechtsschutz im Internet in der nächsten Zeit anstehen. Besonders das Urteil des US Supreme Court zeigt sich hierbei als interessant, da es eine völlig neue Richtung bzw. Möglichkeit der Vorgehensweise eröffnet.

3I Emule kann bei Chip-Online heruntergeladen werden: http://www.chip.de/downloads/ci_downloads_i2990359.html

32 Heise-Online vom 27.06.2005; http://www.heise.de/newsticker/meldung/6 I I 20

33 Spiegel-Online vom i I.04.2005, »Premiere verklagt Softwarehersteller«, http://www.spiegel.de/netzwelt/politik/o, I 5 I 8,35077,, 00 .html

34 Bisher wurde nur eine einstweilige Verfügung erwirkt,jedoch kann das Urteil nicht mehr in die Erörterungen einbezogen werden 